\title{
GLI INVESTIMENTI DIRETTI ESTERI DELLA FEDERAZIONE RUSSA
}

\author{
Francesca Spigarelli ${ }^{*}$
}

\section{Premessa}

Gli investimenti diretti esteri (Ide) rappresentano un tassello fondamentale per la crescita e l'espansione internazionale delle economie emergenti, Brasile, Russia, India e Cina (cosiddetti "Bric") in particolare (Sauvant, 2005, 2008). Aspetti macro e micro economici stanno concorrendo a stimolarne in modo eccezionale le attività transnazionali. Da un lato, gli Ide possono costituire, in una visione-Paese, una leva essenziale per l'aggiustamento di percorsi di crescita fortemente accelerati e spesso squilibrati, soprattutto a fronte dell'eccesso di flussi esportativi e delle pressioni sui tassi di cambio (Oecd, 2008 , p. 80). Al contempo, attraverso la realizzazione di investimenti nelle aree industrializzate si ricerca una rapida affermazione politica ed economica su scala globale. Dall'altro lato, fattori specifici del settore o della singola impresa spiegano l'espansione rilevante delle risorse investite all'estero: accesso a fonti energetiche e materie prime; acquisizione di tecnologia, marchi, competenze, conoscenze, canali distributivi; apertura a nuovi mercati di sbocco (Sauvant, 2005, 2008; Ramamurti e Singh, 2009; Goldstein, 2009).

La Russia è sicuramente una delle nazioni protagoniste in questo scenario. I problemi politici ed economici successivi alla disgregazione dell'Impero avevano messo in secondo piano il Paese rispetto alle altre economie emergenti. Un netto cambiamento di rotta, spinto prevalentemente dal rialzo dei prezzi dei principali beni esportati, ha invece proiettato la Russia nello scenario economico globale a partire dagli anni Novanta (Braguisky e Myerson, 2007; Oecd, 2009). Un segno forte della sua potenza sono divenuti gli investimenti diretti all'estero (Panibratov e Kalotay, 2009, p. 1) che per portata ed intensità, si contraddistinguono rispetto a quelli degli altri Paesi Bric.

*Dipartimento di studi giuridici ed economici, Università di Macerata, spigarelli@unimc.it

QA - Rivista dell'Associazione Rossi-Doria, 1, 2011 
In questo contributo si propone un'analisi delle principali caratteristiche del fenomeno, con un'attenzione specifica anche al contesto italiano. Si intendono indagare intensità degli Ide russi, protagonisti principali e motivazioni sottostanti. Il lavoro si inserisce in un più ampio percorso di ricerca, teso a comparare l'attività di investimento dei Paesi Bric in occidente ed a coglierne $i$ tratti distintivi, in modo sia da elaborare una possibile chiave di lettura del fenomeno stesso nell'ambito delle teorie sugli investimenti internazionali (Buckley, 2002), sia da comprenderne i risvolti di tipo economico e di politica industriale (Spigarelli, 2009). Una particolare attenzione è rivolta, come accennato, al sistema produttivo italiano': l'internazionalizzazione passiva, generata dalle economie emergenti, alimenta, infatti, interessanti spunti di ricerca ed interrogativi anche in relazione al possibile impatto sui suoi sentieri di sviluppo, sulla trasformazione in atto delle aree distrettuali, oltre che sui processi di riconfigurazione della conoscenza e di allocazione del valore in ambito locale.

In questa logica, il paper rappresenta una prima analisi esplorativa del caso russo, volta a rispondere ad alcuni interrogativi che dovrebbero stimolare ulteriormente l'attività di ricerca: quali aspetti, criticità e prospettive connotano attualmente i flussi di investimento e le operazioni realizzate a livello globale dalle imprese russe? Le iniziative in Italia assumono delle peculiarità in termini di settori coinvolti, motivazioni sottostanti, modalità di ingresso? Il loro impatto sul sistema produttivo nazionale, soprattutto in termini prospettici, è rilevante?

Con riferimento alla metodologia di analisi, l'unico approccio percorribile per poter giungere ad alcune considerazioni generalizzabili è risultato quello descrittivo. Il fenomeno degli investimenti esteri russi, analogamente a quanto riscontrabile per gli altri Paesi emergenti, è infatti nuovo, e non sono a tutt'oggi fruibili serie di dati ampie, in grado di supportare analisi quantitative articolate. Tale indisponibilità si sovrappone spesso alla non completa attendibilità o comparabilità temporale dei dati stessi: solo recentemente, infatti, sono stati innovati, da parte di questi Paesi, Russia in primis, i sistemi di rilevazione statistica (Panibratov e Kalotay, 2009; Kuznetsov, 2008).

L'autrice ringrazia due anonimi referee per gli utili commenti, Invitalia (in particolare la dottoressa Muscolo) e la Divisione statistiche sull'Estero della Banca d'Italia per i dati e le elaborazioni messi a disposizione utlizzati in questo lavoro.

1. Nell'ambito del richiamato percorso di ricerca, l'attività si è concentrata, sino ad ora, sul caso della Cina. Si sono analizzati i connotati della politica del Go Global intrapresa dal Governo per stimolare la globalizzazione del proprio tessuto imprenditoriale (Bellabona e Spigarelli, 2007), oltre che le peculiarità dell'attività di investimento delle Mne cinesi (Bellabona e Spigarelli, 2006; Boffa et al., 2008), con un focus sul caso italiano (Spigarelli, 2009). 
Il lavoro è strutturato come segue. Il primo paragrafo inquadra l'analisi dell'attività transnazionale russa nel contesto della letteratura sulle multinazionali (Mne) dai Paesi emergenti. I successivi paragrafi sono concentrati sui connotati dell'attività di investimento dell'economia russa a livello globale ed europeo. Nel quarto paragrafo l'analisi si focalizza, invece, sul caso italiano. Infine, si propongono alcune riflessioni di sintesi su criticità e prospettive del processo d'internazionalizzazione della Federazione, oltre che alcune considerazioni funzionali agli obiettivi del percorso di ricerca di lungo termine accennato in precedenza.

\section{Un inquadramento teorico del fenomeno}

Lo studio delle dinamiche e delle caratteristiche dell'aftività d'investimento russa si ricollega alla nuova letteratura sulle Mne provenienti dai $\mathrm{Pa}$ esi emergenti (Bonaglia et al., 2007; Mathews, 2002, 2006; Bonaglia e Goldstein, 2008; Ramamurti, 2008; Sauvant, 2008)2. Osservando l'attività di questi operatori, che si è intensificata a partire dagli anni Novanta (Aykut e Goldstein, 2006; Unctad, 2006), la dottrina ha riscontrato alcune anomalie e specificità.

Gli aspetti salienti che differenziano il comportamento dei nuovi player internazionali, in raffronto alle multinazionali "classiche" (Dunning et al., 2008, p. 176; Guille'n e Garci'a-Canal, 2009), sono riconducibili, innanzitutto, alla velocità del processo di internazionalizzazione ed alla tipologia di strumenti di ingresso adottati. Le nuove Mne si aprono alla competizione estera in modo accelerato, piuttosto che seguendo un percorso graduale e per step predefiniti come têrizzato dalla Scuola di Uppsala (Johanson e Wiedersheim-Paul, 1975; Johanson e Vahlne, 1977). Le modalità di ingresso prevalenti, ossia le acquisizioni e le alleanze, sono coerenti con l'esigenza di acquisire rapidamente asset strategici quali marchi, tecnologie, competenze (Gammeltoft, 2008). Il cambiamento radicale rispetto al passato riguarda, in questa prospettiva, non solo il prevalere di strategie non greenfield, ma anche il fatto di muoversi nel contesto internazionale per ricercare nuovi vantaggi competitivi, piuttosto che per affermarne di già posseduti. Si passa, dunque, dalla prospettiva di un'internazionalizzazione fina-

2. Per una rassegna analitica delle teorie economiche legate alle determinanti degli investimenti diretti esteri, si veda Valdani e Bertoli (2006, pp. 45-69). Per un esame dei principali filoni teorici sul tema dell'internazionalizzazione, rispetto a cui inquadrare la recente letteratura sulle Mne dai Paesi emergenti, si rinvia a Spigarelli, 2009. In relazione alla letteratura di tipo strategico manageriale, collegata alle Mne delle economie emergenti, si rinvia al lavoro di Yamakawa et al., 2008. 
lizzata a sfruttare competenze e vantaggi consolidati in madre patria (asset exploiting) ad un'apertura all'estero finalizzata alla conquista di nuove risorse, ad alto valore aggiunto, nella logica asset exploring (Cantwell, 1989; Kuemmerle, 1999). Operando in un contesto domestico difficile o comunque politicamente instabile, le nuove Mne possiedono elevate abilità politiche e di negoziazione. Infine, mentre le multinazionali del passato si caratterizzavano per una ridotta adattabilità organizzativa, a causa della presenza di strutture $\mathrm{e}$ cultura radicate, le nuove protagoniste della scena mondiale si rivelano spesso altamente flessibili. Esse fanno proprio leva su innovazioni strategiche ed organizzative per espandersi in modo molto rapido (Mathews, 2002, 2006).

Oltre a questi aspetti, vanno considerati ulteriori elementi, in grado di condizionare a tal punto i pattern di espansione delle Mne dai Paesi emergenti da richiedere una nuova chiave di lettura teorica (Bonaglia e Goldstein, 2008). Si fa riferimento, in particolare, alle rilevanti imperfezioni del mercato dei capitali (Scott, 2002; Morck, Yeung e Zhao, 2008), al possesso di vantaggi di proprietà specifici (Deng, 2003; Cuervo-Cazurra e Genc, 2008; Bertoni et al., 2008), oltre che alla presenza di fattori istituzionali (Meyer e Nguyen, 2005; Wright et al., 2005; Bonaglia e Goldstein, 2008). Le tradizionali teorie non sarebbero utilizzabili per descrivere ed interpretare l'attività delle nuove Mne, sia a livello microeconomico di impresa, sia più in generale per analizzare le direttrici dei flussi di investimento provenienti dalle economie emergenti.

Focalizzando l'attenzione sul caso russo, va rilevato come il tema degli investimenti diretti esteri sia stato oggetto di analisi da parte della dottrina solo di recente. I contributi principali riguardano, in primo luogo, le strategie di espansione internazionale delle imprese (Bulatov, 1998, 2001; Liuhto, 2001a,b; 2005; Liuhto e Jumpponen, 2003; Vahtra e Liuhto, 2004), talvolta con riferimento a settori o comparti specifici (Elenkov, 1995a,b; Heinrich, 2001, 2003, 2005). Altri interessanti studi si concentrano su aspetti manageriali e di business (Kets de Vries et al., 2004; Shekshnia, 2001).

In una prospettiva più macroeconomica, alcuni autori descrivono i problemi di misurazione dei flussi e degli stock di investimento (Gusev, 2004) o raffrontano i trend di investimento russi con quelli globali (Andreff, 2002, 2003). Altri sottolineano la necessità di un adattamento delle teorie "classiche" sugli investimenti diretti esteri per consentire una interpretazione efficace del caso russo (Bevan e Estrin, 2004; Kalotay, 2003, 2005, 2008a). In particolare, sono messi in discussione (Kalotay, 2008a) sia il paradigma di Heckscher-Ohlin-Samuelson (Hos) (Heckscher, 1919; Ohlin, 1933; Samuelson, 1948, 1949) e l'Investment development path (Idp) di Dunning (Dunning, 1981, 1986) sia la Stage theory della scuola di Upssala (Johan- 
son e Wiedersheim-Paul, 1975; Johansson e Vahlne, 1977, 1990) e il paradigma eclettico di Dunning (Dunning, 1977, 1993).

Un'attenzione specifica viene posta proprio al modello di Dunning (Oli paradigm). Seguendo tale paradigma le imprese russe, appartenenti tra l'altro ad un contesto-Paese con un livello di sviluppo, ricchezza e tecnologia inferiore a quelli occidentali, avrebbero dovuto prevalentemente reinvestire nel mercato interno, fino a quando non fossero maturati specifici vantaggi e competitività rispetto ai player internazionali (Dunning, 1988). Le Mne russe hanno, per certi versi, rotto il paradigma ${ }^{3}$, espandendosi a livello internazionale in modo rapido ed inatteso (Kalotay, 2008a), pur non disponendo di tecnologie o capacità manageriali mature. Il processo di privatizzazione ha generato una spinta propulsiva alla loro apertura transnazionale: le ingenti risorse finanziarie a disposizione sono state reinvestite all'estero, per acquisire asset fisici ed intangibili con cui cercare anche di coprirsi dai rischi specifici del mercato interno (Settles, 2009, p. 3$)^{4}$.

$\mathrm{Su}$ questi aspetti si avrà modo di tornare e riflettere nel corso del prossimo paragrafo, commentando i dati sull'attività di investimento estero delle imprese russe.

\section{Gli investimenti diretti all'estero: un quadro a livello globale}

La Russia è attualmente il secondo maggior investitore tra le economie emergenti dopo Hong Kong ${ }^{5}$. Nel periodo 1995-2007 il tasso di crescita dello stock degli investimenti esteri è stato il maggiore tra i Bric (tabella 1), grazie alla rapida e forte espansione della presenza all'estero delle sue imprese multinazionali. Nel 2008 la crisi finanziaria ha pesantemente ridimensionato il valore degli investimenti stessi (Connolly, 2009), analogamente a quanto avvenuto a livello globale per tutti i Paesi, ma il trend di espansione rimane comunque positivo (tabella 2), tant'è che proprio nel 2008 la Federazione ha raggiunto il picco di flussi, con oltre 52 mld Usd. Considerando il valore dei flussi generati annualmente ed i tassi di espan-

3. Per approfondimenti sulla capacità del paradigma di Dunning di interpretare il caso dell'internazionalizzazione delle imprese russe, si rinvia a Spigarelli (in corso di pubblicazione).

4. Si renderebbe in questo senso necessario, per comprendere nella sua interezza il fenomeno degli investimenti diretti russi, estendere il paradigma Oli, aggiungendo ai vantaggi Ownership, Locational e Intenalization, la dimensione home country $(\mathrm{H})$ relativa alle peculiarità del contesto politico ed istituzionale domestico (Kalotay, 2008b, p. 60).

5. Hong Kong, essendo dotata di ampia autonomia, viene tipicamente mantenuta separata dalla Cina nelle statistiche ufficiali. Unendo i dati degli investimenti diretti in uscita delle due aree, la Russia si configura seconda tra i Paesi Bric. 
sione degli stessi, il Paese potrebbe rapidamente trasformarsi in investitore netto. Nel 2008, gli Ide in entrata hanno, infatti, superato i 70 mld Usd, mentre lo stock detenuto da investitori stranieri nel Paese ha superato i 214 mld Usd (Unctad, 2009b). Utilizzando i dati della Bank of Russia, la posizione di investitore netto sarebbe, invece, già stata raggiunta (Kalotay, 2008a).

Tab. 1 - Stock Ofdi (Mld Usd)

\begin{tabular}{lrrrrr}
\hline Paese & \multicolumn{1}{c}{1995} & \multicolumn{1}{c}{2000} & \multicolumn{1}{c}{2005} & \multicolumn{1}{c}{2007} & \multicolumn{1}{c}{2008} \\
\hline Usa & $1.363,8$ & $2.694,0$ & $3.638,0$ & $5.228,0$ & $3.071,2$ \\
Hong Kong & 78,8 & 388,4 & 471,3 & $1.011,2$ & 775,9 \\
Federazione russa & 3,3 & 20,1 & 146,7 & 370,2 & 202,8 \\
Brasile & 44,5 & 51,9 & 79,3 & 136,1 & 162,2 \\
China & 17,8 & 27,8 & 57,2 & 95,8 & 147,9 \\
India & 0,5 & 1,9 & 10,0 & 44,1 & 61,8 \\
\hline
\end{tabular}

Fonte: Panibratov, Kalotay (2009, p. 6).

La crescita considerevole degli investimenti è attribuibile sia all'incremento effettivo dei flussi, sia ai nuovi sistemi di rilevazione dati. Con riferimento a quest'ultimo aspetto, va rilevato come il governo russo abbia recentemente adottato importanti provvedimenti per migliorare il sistema di raccolta e diffusione delle informazioni sugli investimenti diretti. Gli analisti e le istituzioni internazionali sottolineano, infatti, da tempo, il problema della misurazione e si è concordi sul considerare i valori ampiamente sottostimati (Bulatoy, 1998; Kalotay, 2005, 2008b; Kuznetsov, $2008)^{6}$.

Tab. 2 - Flussi Ofdi (Mln Usd)

\begin{tabular}{lccccc}
\hline Paese & $1990-2000($ media $)$ & 2005 & 2006 & 2007 & 2008 \\
\hline Russia & 1.294 & 12.767 & 23.151 & 45.916 & 52.390 \\
Brasile & - & - & 28.202 & 7.067 & 20.457 \\
India & - & - & 14.344 & 17.281 & 17.685 \\
Cina & 30.104 & 72.406 & 21.160 & 22.469 & 52.150 \\
\hline
\end{tabular}

Fonte: Unctad (2009a, pp. 247-250).

Peraltro, la ridotta affidabilità dei dati è aggravata dalla rilevanza del fenomeno del round tripping. Gli investimenti sono realizzati spesso attraverso società che hanno sede in Paesi terzi, per cogliere le agevolazioni, so-

6. Secondo alcune stime, i flussi non registrati dalla Russia nel periodo 1992-2002 sarebbero pari a $245 \mathrm{mld}$ Usd (Unctad, 2005, p. 4). 
prattutto di tipo fiscale, offerte agli operatori stranieri. Testimonianza di ciò sono gli ingenti ammontari di risorse che coinvolgono Cipro (Pelto et al., 2003), Olanda e British Virgin Islands (Kalotay, 2008a; Connolly, 2009) ${ }^{7}$.

Le statistiche appena richiamate consentono alcune riflessioni sull'adeguatezza degli approcci teorici più consolidati al caso russo. In primo luogo, in quanto economia emergente, la Russia dovrebbe caratterizzarsi per scarsità di valuta estera e di capitali ed attirare risorse dai Paesi sviluppati, ricchi di vantaggi competitivi in settori complessi e ad alta intensità di capitale (Kalotay, 2008a). La Federazione dovrebbe trovarsi in una condizione di destinatario di investimenti dall'estero (Sauvant, 2005) ${ }^{8}$. Si è visto, invece, come la stessa si stia affermando quale investitore netto, anche a fronte di un'ampia disponibilità di risorse valutarie, veicolate dagli elevati flussi esportativi. Tale posizione, a seconda della fonte di dati utilizzata, sarebbe già raggiunta $\mathrm{o}$ comunque molto prossima.

In secondo luogo, analogamente a quanto riscontrato per altre economie emergenti (Unctad, 2006; Bonaglia et al., 2007; Goldstein e Pusterla, 2008), l'accentuato livello di internazionalizzazione delle imprese domestiche potrebbe definirsi "prematuro", in raffronto allo stadio di sviluppo della nazione e del sistema economico. Tale fenomeno, che si è notevolmente accentuato negli ultimi anni, renderebbe l'approccio dell'Idp di Dunning ${ }^{9}$ non efficace nell'interpretare il processo dì internazionalizzazione russo (Kalotay, 2008a).

Oltre ai dati relativi ai flussi/stock di investimento, il fenomeno degli Ide russi in comparazione con gli altri Paesi Bric, può essere letto facendo riferimento alle iniziative di tipo greenfield ed alle operazioni di fusione ed acquisizione (Merger and Acquisition - M\&A).

L'apertura ex novo di unità produttive e/o commerciali all'estero (tabella 3) vede la Russia in seconda posizione rispetto ai Paesi emergenti, con tassi di crescita significativi nel periodo 2004-2008 (+72\%). Analogamente,

7. Il fenomeno del roundtripping ha generato, secondo le stime disponibili, un valore di circa 7 mld Usd tra il Gennaio 1997 e il giugno 2008, pari al 10\% del totale degli investimenti russi (Panibratov e Kalotay, 2009, p. 2).

8. La tendenza riscontrata per l'economia russa è analoga a quella di molte economie emergenti che presentano spesso squilibri collegati all'eccesso di disponibilità valutarie per gli elevati flussi di esportazioni. Il caso della Cina, in questo senso, è emblematico (Bellabona, Spigarelli, 2006, 2007). I governi si fanno spesso promotori di correzioni di tali sentieri di crescita squilibrati attraverso politiche di sostegno degli investimenti all'estero. Non solo mediante incentivi fiscali, finanziari ed amministrativi alle imprese, ma anche attraverso la costituzione di veicoli d'investimento specifici a controllo pubblico si stimolano gli investimenti internazionali.

9. Per approfondimenti sull'Idp e sulla sua applicabilità al caso dei Paesi emergenti si rinvia a Goldstein e Pusterla (2008, pp. 14-15). 
l'espansione russa in ambito internazionale è particolarmente forte attraverso fusioni ed acquisizioni: dal 2001 al 2006 la Federazione ha accresciuto di oltre il 300\% il valore degli Ide attraverso questa modalità di ingresso (tabella 4).

Tab. 3 - Progetti di investimento greenfield (numero iniziative completate)

\begin{tabular}{lrrrrrc}
\hline & 2004 & 2005 & 2006 & 2007 & 2008 & 2009 (I trim.) \\
\hline Russia & 109 & 139 & 155 & 135 & 188 & 30 \\
Brasile & 40 & 34 & 39 & 64 & 97 & 16 \\
India & 203 & 192 & 295 & 215 & 345 & 57 \\
Cina & 102 & 99 & 116 & 117 & 161 & 24 \\
\hline
\end{tabular}

Fonte: Unctad (2009a, pp. 212-214).

La prevalenza di strumenti di crescita esterna nel percorso di internazionalizzazione delle Mne russe non è in linea, sul piano teorico, con quanto previsto dalla scuola di Uppsala. Le acquisizioni sono, infatti, uno strumento complesso da governare, che dovrebbe essere prescelto solo a fronte della maturazione di una buona esperienza internazionale. Nel caso russo, esse hanno rappresentato la modalità di espansione preminente sin dalle fasi iniziali di apertura all'estero.

Tab. 4 - Valore M\&A dai Paesi Bric (in mln Usd a prezzi correnti, registrato nel Paese dell'impresa target)

\begin{tabular}{lcccccr}
\hline & 2001 & 2002 & 2003 & 2004 & 2005 & \multicolumn{1}{c}{2006} \\
\hline Russia & $2.038,6$ & $1.251,6$ & $7.879,8$ & $4.061,6$ & $2.819,1$ & $8.676,9$ \\
Brasile & $7.002,9$ & $5.897,4$ & $5.271,1$ & $6.639,2$ & $5.799,7$ & $10.034,6$ \\
India & $1.037,4$ & $1.697,6$ & 948,6 & $1.760,3$ & $4.209,7$ & $6.715,6$ \\
Cina & $2.324,6$ & $2.072,5$ & $3.820,1$ & $6.768,4$ & $8.252,7$ & $6.723,8$ \\
\hline
\end{tabular}

Fonte: elaborazioni proprie su dati Unctad, http://stats.unctad.org/FDI/.

Focalizzando l'attenzione proprio sulle operazioni di fusione ed acquisizione transnazionali ${ }^{10}$, è utile riflettere sulla composizione settoriale degli investimenti (tabella 5), oltre che sulla loro destinazione geografica (tabella 6). Il valore delle $\mathrm{M} \& \mathrm{~A}$ è cresciuto in modo significativo nel periodo gennaio 2005-giugno 2008 rispetto agli anni precedenti. A livello settoriale, la maggioranza delle operazioni ha riguardato le industrie del primario, che hanno

10. Si tratta, come noto, di un dato da utilizzare con cautela considerando che si fa riferimento a transazioni annunciate, il cui perfezionamento in termini finanziari potrebbe riguardare anche il medio periodo o, addirittura, potrebbe non realizzarsi a causa dell'annullamento della transazione stessa. 
catalizzato circa i 2/3 delle risorse investite tra il 1997 ed il 2008. Meccani$\mathrm{ca}$, metalli ed autoveicoli sono, invece, i comparti del manifatturiero più coinvolti in operazioni non greenfield. I servizi sono rimasti meno interessati ad azioni di penetrazione internazionale, che hanno coinvolto, peraltro, in prevalenza le telecomunicazioni.

Tab. 5 - Acquisizioni all'estero delle Mne russe, per settore, gennaio 1992-giugno $2008(\mathrm{mln} U \mathrm{sd})$

\begin{tabular}{lcccc}
\hline Settore & $1992-1996$ & $1997-2000$ & $2001-2004$ & $2005-2008$ \\
\hline Primario & 45 & 1.098 & 2.980 & 33.485 \\
Secondario & 451 & 146 & 661 & 13.430 \\
Servizi & 15 & 456 & 1.857 & 8.935 \\
Totale & 511 & 1.700 & 5.498 & 55.850 \\
\hline
\end{tabular}

Fonte: Panibratov e Kalotay (2009), su dati Unctad (2009a, p. 6).

L'esame dei dati sulle M\&A in termini di distribuzione geografica delle risorse investite (tabella 6) fa emergere un'interessante peculiarità dell'attività di investimento estero russa, che la contraddistingue dagli altri Paesi Bric. Si fa riferimento alla predominanza di destinazioni legate ai Paesi industrializzati dell'occidente, piuttosto che ad aree in via di sviluppo dell'Africa, dell'Asia o dell'America Latina. Europa e Stati Uniti sono i destinatari prioritari delle iniziative di M\&A, dopo i membri del Commonwealth of indipendent states (Cis).

Tab. 6 - Acquisizioni all'estero delle Mne russe, per area geografica di destinazione, gennaio 1992-giugno 2008 ( $m \ln U s d$ )

\begin{tabular}{lcccc}
\hline Paese & $1992-1996$ & $1997-2000$ & $2001-2004$ & $2005-2008$ \\
\hline Europa & 311 & 1.749 & 2.766 & 30.575 \\
Nord America & - & 170 & 1.195 & 13.247 \\
Altri Paesi & & & & \\
industrializzati & 200 & 232 & - & 465 \\
Africa & - & - & - & 250 \\
Asia e Oceania & - & - & - & 2.945 \\
Europa Est e Cis & - & 61 & 1.536 & 9.297 \\
Totale & 511 & 2.212 & 5.497 & 56.779 \\
\hline
\end{tabular}

Fonte: Panibratov e Kalotay, 2009, p. 7.

Nei primi, le imprese russe possono aver accesso a mercati di sbocco ed a risorse strategiche necessarie per la loro affermazione su scala globale, come verrà meglio esaminato di seguito.

Con riferimento al Cis, invece, va sottolineato come questi Stati, ed in 
particolare Ucraina, Kazakhistan e Bielorussia ${ }^{11}$, abbiano rappresentato e rappresentino tuttora una prima, naturale, via di internazionalizzazione per le imprese russe (Liuhto e Jumpponen, 2003). In tali aree, culturalmente affini e geograficamente vicine, è possibile avere accesso a vasti mercati di sbocco, assumendo posizioni di leadership. Inoltre, vi è ampia disponibilità di risorse naturali. Ciò rende il Cis una fonte di grande interesse per gli investitori russi, particolarmente attivi nel primario.

Coerentemente con quanto previsto dalla scuola di Uppsala, la scelta dei Paesi di destinazione ha dapprima favorito queste aree geografiche, culturalmente vicine, per poi coinvolgere i Paesi occidentali più evoluti (Kalotay, 2008a). In ogni modo, nel riscontrare la forte presenza russa nel Cis non vanno dimenticate le ragioni economiche e politiche legate al periodo di transizione, successivo alla disgregazione dell'Impero russo, quando, a fronte della totalità di imprese manifatturiere in mano pubblica, si è passati ad una frammentazione della proprietà delle imprese stesse nei vari territori indipendenti. Per risolvere i problemi di coordinamento tra aziende ormai formalmente separate, ma sostanzialmente collegate da rapporti produttivi e commerciali, le imprese russe hanno avviato numerose operazioni di M\&A. L'acquisizione è stata lo strumento più immediato ed efficace per riprendere il controllo delle "filiali" dislocate nel Cis (Kalotay, 2008a, pp. 10-11).

Altro aspetto interessante da esaminare, per inquadrare l'attività di investimento su scala globale della Federazione, riguarda i suoi "protagonisti". Si tratta tipicamente di soggetti a controllo pubblico o di grandi imprese privatizzate, frutto del processo di trasformazione dell'economia verso un modello capitalistico che ha coinvolto in prevalenza investitori interni (Kalotay, 2001; Locatelli, 2006; Kets de Vries et al., 2004). Gazprom, Lukoil, Surgutneftegas, Rosfnet Oil, Sberbank, Rusal, Evraz, Norilsk Nickel, Russian Alluminium, Severstal, Youkos, Gmz sono i principali player russi (Kalotay, 2008a) $)^{12}$. Le industrie in cui essi operano sono essenzialmente quelle del petrolio e gas, della metallurgia, della finanza, delle telecomunicazioni (Kuznetsov, 2008), tutte caratterizzate ancora da forti interessi ed ingerenze dello stato (Liuhto, 2007). Più recentemente, nuovi protagonisti hanno cominciato ad affermarsi sia nel settore tradizionale delle risorse energetiche e delle materie prime (ChTPZ Group, Koks, Metalloinvest, Magnitogorsk Iron \& Steel Works), sia in industrie che nel passato erano com-

11. Analizzando i flussi di investimento esteri russi Kuznetsov (2008) evidenzia come il $30 \%$ degli stessi sia indirizzato verso il Cis e di questo circa l' $80 \%$ sia diretto verso Ucraina, Kazakhstan, Bielorussia.

12. Nell'elenco Fortune global 500 di fine 2008 sono 47 le Mne dai Paesi Bric. Di queste, 5 sono russe (Gazprom, Lukoil, Surgutneftegas, Rosfnet Oil, Sberbank). Gazprom è al $47^{\circ}$ posto, quarta tra le Mne dai Bric dopo 3 imprese cinesi. 
pletamente chiuse a percorsi di internazionalizzazione (Kuznetsov, 2008). $\mathrm{Si}$ fa riferimento al comparto dei macchinari (Rostelmash, Transmash holding), della carta (Investlesprom), dell'alimentare (Russian Solod, SPI gruop, Russian Vine Trust, Wimm-Bill-Dann, Mezhrespublikanskij Vinzavod, Natiusha), della profumeria (Kalina).

La tabella 7 evidenzia il valore degli asset fissi investiti all'estero per le prime 30 Mne russe.

Le motivazioni che guidano gli investimenti sono da ricondursi a strategie sia di "esodo", sia di "espansione attiva".

Soprattutto nelle fasi iniziali di apertura dell'economia russa, gli Ide sono stati per le imprese uno strumento per trovare un ambiente di business più sicuro e favorevole rispetto a quello domestico (Guille'n e Garc1'a-Canal, 2009), fortemente incerto e con scarsa tutela della proprietà (Sauvant, 2005; Kalotay, 2002; Bulatov, 1998). Tutt'ora per molte impresê questa finalità risulta prevalente: si realizzano operazioni transnazionali per rafforzare l'immagine interna ed aumentare il potere negoziale in madrepatria $(\mathrm{Ku}-$ znetsov, 2008). Sembrerebbe, in questa logica, ricollegabile al caso russo quanto previsto dalla teoria dello squilibrio (Moon e Rohel, 1993; 2001) per lo stimolo offerto ai processi di internazionalizzazione dagli svantaggi competitivi del Paese di origine.

Più recentemente, sono invece emerse anche motivazioni sintomatiche di un atteggiamento più "attivo" da parte degli investitori, legate alla conquista di risorse ed asset strategici fondamentali per operare sui mercati occidentali (Liuhto, 2005; Kheifets, 2008). Si ricerca, in specie nel settore primario, il controllo più esteso possibile della catena del valore. Gli investimenti rispondono, dunque, tipicamente a finalità di tipo efficiency seeking. Nell'industria petrolifera e del gas, le aziende russe acquisiscono, a monte, raffinerie per la lavorazione della materia prima. Nel contempo, a valle della filiera, le acquisizioni riguardano catene distributive, gasdotti e linee di distribuzione del greggio, reti distoccaggio, oltre che stazioni di rifornimento carburante. In tal modo, si tende ad avere accesso ad una quota elevata del valore generato nel processo produttivo e distributivo, oltre che a controllare in modo più esteso la domanda estera (Sauvant, 2005), raggiungendo direttamente il consumatore finale (Connolly, 2009). L'approvvigionamento di fonti ulteriori di risorse naturali quali petrolio, metalli o minerali spinge, inoltre, le imprese del primario ad acquisizioni anche nella logica resource seeking.

In questo senso, l'atteggiamento delle Mne russe appare coerente con quanto teorizzato dal paradigma eclettico di Dunning. L'aspetto più evidente riguarda i vantaggi di internalizzazione, in relazione alla tendenza degli investitori russi a ricercare il controllo più ampio possibile di attività com- 
Tab. 7 - Le prime 30 Mne russe per asset fissi investiti all'estero (valori in mld Usd, fine 2007)

\begin{tabular}{lll}
\hline $\begin{array}{l}\text { Asset fissi } \\
\text { investiti }\end{array}$ & \multicolumn{1}{c}{ Azienda } & \multicolumn{1}{c}{ Principale attività svolta } \\
\hline$>13,0$ & Lukoil & Estrazione, lavorazione e distribuzione petrolio; \\
chimica
\end{tabular}

Fonte: Kuznetsov, 2008, p. 6.

plementari e collegate al proprio core business. Ciò per affermare, anche all'estero, vantaggi di proprietà posseduti (Sauvant, 2005, p. 652), soprattutto nella logica dei vantaggi di transazione ${ }^{13}$ : l'abilità maggiore delle im-

13. In particolare, si fa riferimento al possesso di vantaggi di transazione (fattore "Ot"), nel modello rivisto di Dunning (Dunning e Lundan, 2008). Si sfrutterebbero, infatti, vantaggi di proprietà (ownership advantage) di tipo "Ot" legati al possesso di esperienze e compe- 
prese russe si riscontrerebbe, infatti, proprio nella gestione della catena del valore (Kalotay, 2008a). In questa logica, un modello interessante da richiamare per interpretare efficacemente la situazione russa fa riferimento alla letteratura di international business e alla presenza di fattori sia firm specific, sia country specific nello spiegare il sentiero d'internazionalizzazione delle Mne (Rugman, 2008, pp. 91-95). Nel caso russo, assolutamente peculiare, le imprese sembrano spingersi all'estero per sfruttare vantaggi dei Paesi ospitanti (host country specific advantages), nel settore delle materie prime. Esse tendono ad affermarsi ulteriormente proprio in un ambito in cui godono di vantaggi country specific rilevanti, collegati tipicamente al possesso di risorse energetiche. Il tutto mantenendo il controllo sui vantaggi d'impresa, collegati alle richiamate capacità di coordinamento della propria catena del valore (Rugman, 2008, p. 104).

Sempre più rilevanti nei percorsi di internazionalizzazione divengono, inoltre, gli scopi legati alla conquista di nuovi sbocchi commerciali per i prodotti russi. Molto attivi in questo senso risultano i principali operatori della telefonia mobile della Federazione, con rilevanti operazioni nell'area del $\mathrm{Cis}^{14}$, oltre che le imprese del settore finanziario (Connolly, 2009).

Altre motivazioni che guidano l'espansione delle Mne russe sono riconducibili alla ricerca di risorse strategiche. Esse coinvolgono, tra l'altro, i servizi infrastrutturali all'estero o il superamento di barriere all'operatività in settori protetti. Si pensi, ad esempio, all'industria dell'acciaio negli Usa, caratterizzata da contingentamento nelle quote dí importazioni ${ }^{15}$. Sempre più rilevanti risultano, inoltre, le innovazioni tecnologiche, oltre che le competenze di marketing e management, a cui le imprese russe fanno crescente attenzione considerando il ruolo fondamentale che tali risorse assumono per la penetrazione dei mercati occidentali.

Da ultimo, ma non certamente per ordine di importanza, va citata l'affermazione politica ed economica del Paese su scala globale, quale motivazione degli Ide russi. Il ruolo dello Stato in molte operazioni è ancora forte e centrale. Muovendosi attraverso le cosiddette Patriots (Vahtra e Liuhto, 2004) o Kremlin Universe (Aton corporation, 2004), lo Stato promuove la propria affermazione politica mediante iniziative economiche di

tenze organizzative, piuttosto che a vantaggi "Oa" legati alla disponibilità di asset intangibili. Per un'ampia analisi del paradigma eclettico di Dunning e del suo collegamento con le più recenti teorie sulle Mne, si rinvia a Li et al., 2005.

14. Esempi rilevanti sono le acquisizioni da parte di Mts del $74 \%$ del principale operatore dell'Uzbekistan (Uzdunorbita) e della VimpelCom di uno dei maggiori operatori del Kazakhstan (KaR-Tel).

15. Si consideri, in questo senso, l'acquisizione dell'azienda statunitense Rouge Industries da parte di Severstal, per il superamento delle barriere di accesso al mercato dell'acciaio Usa, dovute alle quote di importazione. 
larga portata, in termini sia finanziari, sia strategici, mirando all'acquisizione del controllo di importanti asset distributivi e produttivi occidentali. L'elevata interferenza del governo avrebbe almeno due rilevanti implicazioni, sul piano teorico. Da un lato, essa contribuirebbe, insieme ai fattori richiamati in precedenza, a rendere non coerente il caso russo a quanto descritto dall'Idp di Dunning. Dall'altro lato, essa renderebbe imprescindibile considerare, nella lettura dei trend di investimento estero, le regole del gioco, formali ed informali, dettate dal contesto istituzionale di riferimento, come suggerito dalla letteratura emergente della institution based view of strategy (North, 1990; Peng, 2002).

\section{I progetti di investimento a livello Europeo}

Dopo aver illustrato l'andamento e le caratteristiche principali dell'attività di investimento estera della Federazione russa a livello mondiale, l'attenzione si sposta in ambito europeo.

L'analisi del valore delle risorse fatte affluire in Europa, in raffronto agli altri Paesi Bric, mostra come la Russia sia sicuramente un partner rilevante per gli stati membri dell'UE (tabella 8). In termini di crescita degli stock investiti, nel periodo 2004-2006, la Russia rappresenta il quinto partner dell'Unione dopo Turchia, Brasile, Norvegia (Eurostat, 2008, p. 44).

L'oscillazione dei flussi che si riscontra nel periodo osservato va attribuita prevalentemente all'impatto delle singole operazioni di investimento. Le iniziative russe si caratterizzano, infatti, per la loro ampia portata finanziaria ed una singola azione incide pesantemente sui valori di stock e flusso nell'anno di riferimento.

Tab. 8 - Investimenti diretti esteri dei Paesi Bric in Europa (flussi, in mld euro)

\begin{tabular}{lcccc}
\hline & 2004 & 2005 & 2006 & 2007 \\
\hline Flussi da Paesi Extra UE & 58,3 & 127,0 & 168,9 & 319,2 \\
Bric & 9,6 & 7,2 & 5,2 & 10,4 \\
Russia & 0,3 & 2,8 & 1,5 & 1,0 \\
Brasile & 4,0 & 2,9 & 1,2 & 1,9 \\
India & 0,0 & 0,5 & 0,5 & 9,5 \\
Cina & 5,3 & 1,0 & 2,0 & $-1,9$ \\
\hline
\end{tabular}

Fonte: Petridou, 2008, p. 7.

Rispetto alla lettura dei dati macroeconomici sugli Ide, un fenomeno particolarmente interessante da analizzare riguarda $\mathrm{i}$ progetti d'investi- 
mento greenfield ed espansioni annunciati sulla stampa internazionale ${ }^{16}$. Nell'intervallo temporale tra il 2004 ed il novembre 2009 sono stati presentati 110 progetti d'investimento verso l'Europa da parte di 66 società russe (tabella 9). Il 63\% delle iniziative si è concentrato nei settori dei servizi finanziari, dell'Ict e dell'energia.

In termini di valore degli investimenti ${ }^{17}$, il comparto energetico e quello dei trasporti emergono come dominanti, con una media di capitale investito molto elevato ed una fortissima concentrazione di operazioni. Pochi grandi progetti catturano, nella sostanza, la maggioranza delle risorse fatte affluire dalla Federazione (tabella 10).

L'energia è il settore predominante, con ingenti operazioni realizzate da Gazprom, Yukos, Novatek, Russneft. Mosca è la principale sede ed origine degli investimenti, con quasi il 70\% del totale. L' $8 \%$ proviene da San Pietroburgo, mentre il restante valore è frammentato nelle iniziative di 22 città periferiche, ciascuna con 1-2 progetti presentati nel periodo.

Tab. 9 - Progetti d'investimento annunciati verso l'Europa da parte di società russe per settore di riferimento (numero progetti)

\begin{tabular}{lccccccc}
\hline Settore & 2004 & 2005 & 2006 & 2007 & 2008 & 2009 & Totale \\
\hline Servizi finanziari & 4 & 5 & 5 & 6 & - & 3 & 23 \\
Ict \& Elettronica & 5 & 4 & 2 & 4 & 5 & 3 & 23 \\
Energia & 1 & 4 & 4 & 4 & 4 & 6 & 23 \\
Chimica, plastica, metalli, minerali & 2 & 2 & - & 2 & 2 & 3 & 11 \\
Industrie ludiche e creative & 1 & 3 & 1 & - & - & - & 5 \\
Trasporti, logistica & - & 1 & 2 & 1 & 1 & 1 & 6 \\
Alimentari, bevande, tabacco & 1 & 1 & - & 1 & - & - & 3 \\
Servizi professionali & - & - & 1 & - & 2 & - & 3 \\
Componenti trasporti, automotive & - & - & 1 & 1 & 1 & - & 3 \\
Costruzioni & 2 & - & - & - & - & 1 & 3 \\
Servizi di supporto all'industria & 1 & - & 1 & - & - & - & 2 \\
Turismo & - & - & - & 1 & 1 & - & 2 \\
Beni consumo (elettronica, giocattoli ...) & - & - & - & 1 & - & - & 1 \\
Tecnologia dell'ambiente & - & - & - & - & - & 1 & 1 \\
Sanità & 1 & - & - & - & - & - & 1 \\
Totale & 18 & 20 & 17 & 21 & 16 & 18 & 110 \\
\hline
\end{tabular}

Fonte: nostre elaborazioni su dati Invitalia.

16. I dati presentati sono stati elaborati da parte di Invitalia su informazioni tratte dal data base fDi intelligence, del Financial Times (http://www.fdiintelligence.com). Invitalia, è l'Agenzia nazionale per l'attrazione degli investimenti e lo sviluppo d'impresa, il cui azionista unico è il Ministero dell'economia e delle finanze (www.invitalia.it).

17. L'ammontare investito non è tracciato per tutti i progetti, in quanto il dato non è sempre disponibile. Si tratta di un valore generalmente stimato. 
Tab. 10 - Progetti d'investimento annunciati verso l'Europa da parte di società russe per settore di riferimento (valore progetti - Capex ${ }^{*}$ - in mln Usd)

\begin{tabular}{|c|c|c|c|c|c|c|c|}
\hline Settore & 2004 & 2005 & 2006 & 2007 & 2008 & 2009 & Totale \\
\hline Energia & 30,0 & $6.093,4$ & 323,2 & $1.265,6$ & 801,5 & $2.157,4$ & $10.671,1$ \\
\hline Trasporti, logistica, stoccaggio & - & 36,5 & 121,2 & 15,0 & $1.254,2$ & 15,0 & $1.441,9$ \\
\hline Turismo & - & - & - & 300,0 & 3,3 & - & 303,3 \\
\hline Costruzioni & 125,7 & - & - & - & - & 588,9 & 714,6 \\
\hline Ict \& Elettronica & 34,6 & 184,0 & 11,4 & 90,8 & 106,9 & 18,1 & 445,8 \\
\hline Commercio & - & - & - & - & - & - & - \\
\hline Servizi finanziari & 96,2 & 113,7 & 114,0 & 127,9 & - & 73,5 & 525,3 \\
\hline Tecnologia dell'ambiente & - & - & - & - & - & 18,8 & 18,8 \\
\hline Alimentari, bevande, tabacco & 4,0 & 11,1 & - & 7,1 & - & - & 22,2 \\
\hline $\begin{array}{l}\text { Chimica, plastica, } \\
\text { metalli, minerali }\end{array}$ & 7,3 & 21,6 & - & 12,3 & 211,7 & 70,2 & 323,1 \\
\hline Materiali di trasporto & - & - & 15,2 & 9,0 & 35,0 & - & 59,2 \\
\hline Servizi professionali & - & - & 7,1 & - & 45,0 & - & 52,1 \\
\hline $\begin{array}{l}\text { Media, TV, tempo libero, } \\
\text { editoria, pubblicità }\end{array}$ & 6,2 & 31,3 & 5,6 & - & & - & 43,1 \\
\hline $\begin{array}{l}\text { Beni di consumo } \\
\text { (elettronica, gioielleria, ecc.) }\end{array}$ & - & - & - & 16,6 & & - & 16,6 \\
\hline Settori industriali diversi & 5,9 & - & 5,7 & & & - & 11,6 \\
\hline Sanità e servizi collegati & 8,6 & - & - & & 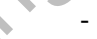 & - & 8,6 \\
\hline Totale & 318,5 & $6.491,6$ & 603,4 & $1.844,3$ & $2.457,6$ & $2.941,9$ & $14.657,3$ \\
\hline
\end{tabular}

* Capex (Capital expenditure) individua le spese per capitale ossia i fondi che un'impresa impiega per acquistare asset durevoli, come ad esempio i macchinari. Si tratta prevalentemente di investimenti in conto capitale che dovrebbero permettere all'azienda di espandere o migliorare la propria capacità produttiva.

Fonte: nostre elaborazioni su dati Invitalia.

In relazione alla finalità, prevale la ricerca di nuovi mercati di sbocco se si analizzano i progetti in termini numerici. Nel $40 \%$ dei casi si tratta, infatti, della costituzione dì uffici commerciali o di unità dedicate alla cura dei servizi di marketing ed assistenza vendita e post vendita per il gruppo di riferimento. Nella prospettiva del valore dei progetti stessi, invece, sono predominanti le motivazioni connesse alla ricerca di maggiore efficienza nell'ambito della propria catena del valore e di risorse strategiche: il $76 \%$ delle iniziative riguarda, infatti, l'acquisizione di asset collegati a logistica e distribuzione. La realizzazione di impianti di distribuzione di gas catalizza la maggioranza degli investimenti greenfield russi in Europa.

A livello geografico, la Germania e la Gran Bretagna sono le principali destinazioni dei progetti. Questi Paesi assorbono, infatti, rispettivamente il $32 \%$ ed il 19\% del numero di operazioni greenfield (tabella 11) realizzate da 23 e da 18 aziende russe. In termini di valore, la Germania ha assorbito nel periodo considerato il $66 \%$ delle risorse, per un valore di $9.698,68 \mathrm{mln}$ 
Usd $^{18}$. Seguono Finlandia ${ }^{19}(1.275,70 \mathrm{mln}$ Usd) e Gran Bretagna $(1.129,50$ mln Usd).

Tab. 11 - Progetti d'investimento annunciati verso l'Europa da parte di società russe per area di destinazione (numero progetti)

\begin{tabular}{lccccccc}
\hline Paese & 2004 & 2005 & 2006 & 2007 & 2008 & 2009 & Totale \\
\hline Germania & 9 & 2 & 3 & 8 & 5 & 6 & 35 \\
Gran Bretagna & 3 & 3 & 7 & 4 & 2 & 2 & 21 \\
Svizzera & 1 & 2 & 1 & 5 & 1 & 1 & 11 \\
Francia & 2 & 2 & 2 & 2 & - & 1 & 9 \\
Svezia & 2 & 3 & - & 1 & - & & 6 \\
Italia & - & - & - & - & 2 & 3 & 5 \\
Olanda & - & 2 & 1 & - & & - & 3 \\
Spagna & - & 1 & - & - & 3 & - & 4 \\
Finlandia & - & 1 & 1 & - & 1 & - & 3 \\
Austria & 1 & 1 & 1 & - & - & 1 & 4 \\
Belgio & - & 1 & - & 1 & - & 1 & 3 \\
Grecia & - & 1 & - & - & - & 2 & 3 \\
Irlanda & - & 1 & - & - & 1 & - & 2 \\
Danimarca & - & - & - & 1 & - & - & - \\
Islanda & - & - & 1 & - & - & - & 1 \\
Totale & 18 & 20 & 17 & 23 & 13 & 7 & 110 \\
\hline
\end{tabular}

Fonte: nostre elaborazioni su dati Invitalia.

Con riferimento agli attori principali delle attività di investimento, a fronte della prevalenza delle industrie del primario e dei progetti nell'energia, Gazprom è l'azienda leader (tabella 12), primo investitore in Europa, con 18 progetti corrispondenti a 9.967,48 mld Usd di valore (68\% del totale affluito nel periodo). Il fenomeno della forte concentrazione a livello geografico e territoriale si riflette, dunque, anche in termini di principali player delle iniziative. Le attivita delle prime 10 imprese catalizzano il $44 \%$ dei progetti ed il $92 \%$ del capitale investito all'estero. Il resto delle iniziative ( $56 \%$ del numero e $20 \%$ del capitale) è frammentato tra 61 società con $1 \mathrm{o}$ 2 progetti ciascuna, a capitale investito molto basso.

18. La Germania rappresenta, in generale, una delle destinazioni preferenziali degli investitori russi, non solo nei tradizionali settori del petrolio e del gas. Cfr. Connolly (2009, pp. 8-9).

19. Il valore elevato di investimenti in Finlandia è collegato all'operazione della Russian Railways per oltre 1mld Usd, nel settore dei trasporti, nel 2008. 
Tab. 12 - I protagonisti degli investimenti (Capex ${ }^{*}-$ in mln Usd)

\begin{tabular}{|c|c|c|c|c|c|c|c|}
\hline & 2004 & 2005 & 2006 & 2007 & 2008 & 2009 & Totale \\
\hline Gazprom & - & $6.057,0$ & 346,4 & $1.264,5$ & 801,5 & $1.498,1$ & $9.967,5$ \\
\hline $\begin{array}{l}\text { Russian } \\
\text { Railways }\end{array}$ & - & - & 15,9 & 15,0 & $1.254,2$ & - & $1.285,1$ \\
\hline Mirax Group & - & - & - & 300,0 & 45,0 & 588,9 & 933,9 \\
\hline Renova & - & - & - & - & - & 531,3 & 531,3 \\
\hline Kaspersky Lab & 3,0 & 20,1 & - & 73,3 & 100,7 & 18,1 & 215,2 \\
\hline Severstal & - & - & - & - & 169,9 & 2,3 & 172,2 \\
\hline Golden Telecom & - & 144,7 & - & - & - & - & 144,7 \\
\hline $\begin{array}{l}\text { Burgas- } \\
\text { Alexandr. }\end{array}$ & - & - & & - & - & 110,4 & 110,4 \\
\hline AirBridge Cargo & - & - & 105,3 & - & - & - & 105,3 \\
\hline Stroimontage & 85,0 & - & - & - & - & - & 85,0 \\
\hline Altre aziende & 230,5 & 269,8 & 135,8 & 191,5 & 86,3 & 192,8 & $1.106,7$ \\
\hline Totale & 318,5 & $6.491,0$ & 603,4 & $1.844,3$ & $2.457,6$ & $2.941,9$ & $14.657,3$ \\
\hline
\end{tabular}

* Capex - vedi tab. 10.

Fonte: nostre elaborazioni su dati Invitalia.

\section{Gli investimenti in Italia}

Dall'analisi dei connotati delle attività di investimento mondiali ed europee della Russia, il focus si sposta sull'Italia. Una prima fonte di informazioni utilizzabile è legata alle rileyazioni della Banca d'Italia, secondo la logica statistica della bilancia dei pagamenti ${ }^{20}$.

Il trend di crescita degli investimenti russi è assolutamente rilevante. Isolando gli effetti della crisi finanziaria che hanno portato nel 2008 ad una decelerazione mondiale dei tassi di espansione degli Ide, l'incremento dei flussi dal 2005 al 2007 è molto forte. Si è passati da circa 3 milioni di euro ad oltre 80 milioni. Il fenomeno è, quindi, in rapida evoluzione ed estremamente dinamico.

A livello geografico, fatto salvo nel 2007 un investimento anomalo ed ingente in Friuli Venezia Giulia nel settore "Famiglie", le regioni privilegiate dalle attività russe sono Toscana, Emilia Romagna, Veneto e Lombardia (tabella 13). Queste quattro aree assorbono nel 2008 oltre il $90 \%$ delle risorse affluite nel Paese.

20. Questo dato, come sottolineato dalla letteratura (Mori e Rolli, 1998; Lipsey, 2001; Federico e Minerva, 2007), risulta non significativo se preso in modo asettico ed isolato, considerando i problemi di tempestività ed esaustività nelle rilevazioni. Tuttavia, esso può offrire alcuni interessanti spunti di riflessione. 
Tab. 13 - Investimenti diretti esteri della Russia in Italia per Regione (flussi in mgl euro)

\begin{tabular}{lrrrrr}
\hline Regione & 2005 & 2006 & \multicolumn{1}{c}{2007} & 2008 & Totale \\
\hline Friuli Venezia Giulia & - & 90 & 50.250 & 300 & 50.640 \\
Toscana & 720 & 749 & 3.738 & 25.270 & 30.477 \\
Emilia Romagna & 450 & 485 & 11.552 & 11.000 & 23.487 \\
Veneto & 83 & 1.023 & 905 & 20.757 & 22.768 \\
Lombardia & 632 & 4.909 & 6.877 & 6.612 & 19.030 \\
Zona non classificata & 516 & 3.832 & 66 & 56 & 4.470 \\
Piemonte & - & 41 & 4.075 & 120 & 4.236 \\
Lazio & 326 & 804 & 1.838 & 549 & 3.517 \\
Liguria & 600 & & 906 & 980 & 2.486 \\
Sardegna & - & 629 & 1.269 & 163 & 2.061 \\
Sicilia & - & - & - & 1.170 & 1.170 \\
Calabria & - & 15 & 260 & 381 & 656 \\
Umbria & - & 60 & 78 & 400 & 538 \\
Abruzzi & - & 150 & - & 379 & 529 \\
Trentino Alto Adige & 15 & - & 20 & 422 & 457 \\
Valle D'Aosta & - & - & - & 400 & 400 \\
Campania & - & 95 & 120 & 20 & 235 \\
Marche & - & - & - & 30 & 30 \\
Puglia & - & - & 20 & - & 20 \\
Totale & 3.342 & 12.882 & 81.974 & 69.009 & 167.207 \\
\hline
\end{tabular}

Fonte: nostre elaborazioni su dati Banca d'Italia.

In termini settoriali, i problemi di rilevazione sono evidenti, dato che la classe delle "Famiglie" assume il peso maggiore (tabella 14). Seguendo la definizione di contabilità nazionale, tale voce comprende gli artigiani, le ditte individuali ed i consumatori. Il contenuto della posta, così fortemente eterogeneo, non consente un esame significativo della reale destinazione degli investimenti su scala nazionale. In ogni modo, a parte questo ambito, risultano preminenti $i$ flussi diretti verso i servizi finanziari e di tipo commerciale, i mezzi di trasporto, la meccanica: nel 2008 essi hanno catalizzato 1'80\% delle risorse affluite dalla Federazione.

Per meglio comprendere i connotati dell'attività di investimento russo in Italia, è utile passare da una visione macro, come quella tracciata dalle rilevazioni della Banca d'Italia, ad un quadro più analitico, basato sulla mappatura delle imprese a capitale russo costituite o acquisite in Italia. Come sottolineato in letteratura, tale tipo di analisi è fondamentale per catturare concretamente la politica di espansione internazionale di un Paese (Cantwell e Barnard, 2008).

In questa logica, a livello temporale, è possibile ricondurre la presenza di imprese della Federazione in Italia a tre fasi principali, corrispondenti a tre diversi periodi storici. La prima, definibile "pionieristica", risale agli 
anni Sessanta, con la comparsa dei primi Uffici di rappresentanza di alcune imprese russe. Esempi ne sono Aeroflot nel 1965 e Vnesheconombank nel 1989. Una seconda fase si avvia, invece, a partire dal 2000 e si caratterizza per l'arrivo di imprese del settore energetico e metallurgico (Severstal, Rusal, Evraz, Renova) alla ricerca tipicamente di risorse naturali ed asset distributivi, analogamente a quanto riscontrato a livello mondiale. L'ultima fase, quella più recente ed in corso, è segnata da una maturazione delle motivazioni degli investimenti, oltre che dalla comparsa di nuovi protagonisti. Si riscontrano, infatti, iniziative volte all'acquisizione di asset strategici tra cui know how, tecnologie e competenze, anche in settori diversi dall'energetico.

Tab. 14 - Investimenti diretti esteri della Russia in Italia per settore (importi in $\mathrm{mgl}$ euro)

\begin{tabular}{|c|c|c|c|c|c|}
\hline Settore & 2005 & 2006 & 2007 & 2008 & Totale \\
\hline Famiglie & 214 & 2.015 & 58.434 & 3.850 & 64.513 \\
\hline inanziarie & - & & 147 & 23.125 & 23.688 \\
\hline lezzi di trasporto & - & & - & 20.316 & 20.316 \\
\hline Yacchine agricole ed in & 300 & & 15.500 & - & 15.800 \\
\hline $\begin{array}{l}\text { Servizi eel commercio, recuperi e ripara- } \\
\text { zioni }\end{array}$ & & & 3.622 & 11.541 & 15.542 \\
\hline ltri servizi destinabili alla vendita & & 1.439 & 1.920 & 4.325 & 8.074 \\
\hline lines & & 3.416 & - & 2.355 & 5.771 \\
\hline ltre bran & 160 & 3.412 & 1.949 & 112 & 5.633 \\
\hline Edilizia & 774 & 1.652 & 262 & 2.534 & 5.222 \\
\hline Servizi d & - & - & - & 795 & 795 \\
\hline $\begin{array}{l}\text { Prodotti in metallo esclusi veicoli di tra- } \\
\text { sporto }\end{array}$ & 600 & - & 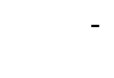 & - & 600 \\
\hline Prodotti chimici & 516 & - & - & 56 & 572 \\
\hline $\begin{array}{l}\text { Minerali e prodotti a base di minerali non } \\
\text { metal. }\end{array}$ & & 357 & - & - & 357 \\
\hline ali & 170 & - & 62 & - & 232 \\
\hline $\begin{array}{l}\text { Prodotti tessili cuoio calzature abbiglia- } \\
\text { mento }\end{array}$ & 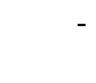 & 14 & 33 & - & 47 \\
\hline Tete & - & - & 2. & - & 25 \\
\hline Oolaziould & - & 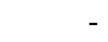 & 20 & - & 20 \\
\hline Totale & 3.342 & 12.882 & 81.974 & 69.009 & 167.207 \\
\hline
\end{tabular}

Fonte: nostre elaborazioni su dati Banca d'Italia.

Conseguentemente a queste tre fasi, risultano ad oggi presenti nel Paese 41 imprese italiane a partecipazione russa ${ }^{21}$. Il $60 \%$ di esse è a possesso totalitario

21. L'analisi degli investimenti russi in Italia è realizzata su dati Invitalia (2009), elaborati dal data base R\&P-Politecnico Milano ed aggiornati a settembre 2009. 
o di controllo, mentre il 10\% è a partecipazione paritaria ed il $30 \%$ a partecipazione minoritaria. Gli addetti occupati sono circa 7.900, per un fatturato complessivo di 4,7 mld di euro. In prevalenza, le imprese sono state oggetto di acquisizione (74\% dei casi), piuttosto che essere costituite ex novo mediante iniziative greenfield.

In termini geografici, gli investimenti si concentrano maggiormente in Lombardia (13 imprese), Emilia Romagna (9 imprese) e Veneto (6 impre$\mathrm{se})^{22}$. Oltre che nelle aree più industrializzate del Paese, la Russia è comunque presente anche nelle Regioni del Sud, che offrono o risorse naturali, come nel caso della Sardegna e della Puglia, o reti infrastrutturali/logistiche/distributive strategiche, come avviene in Sicilia.

Meccanica, metallurgia ed energia sono i settori di principale interesse per gli investitori. Più del $50 \%$ delle imprese controllate opera nell'industria degli strumenti e apparecchiature meccaniche, oltre che nella metallurgia, acciaio in particolare, dove è forte la presenza dei principali player internazionali della Federazione. Intenso è anche l'interesse per l'energia rinnovabile e per le apparecchiature e gli impianti ad essa collegati.

I gruppi Renova e Severstal sono i maggiori investitori, controllando 24 imprese e caratterizzandosi per una forte diversificazione delle aree di investimento e dei settori di interesse (tabella 15).

Tab. 15 - Investimenti non greenfield (NG) e greenfield ( $G$ in Italia di società/gruppi russi (n. di imprese)

\begin{tabular}{lccccc}
\hline Renova & $N G$ & $G$ & Altre imprese & $N G$ & $G$ \\
\hline Energia & 3 & - & Energia & 1 & - \\
Meccanica & 9 & - & Meccanica & 3 & - \\
Metallurgia & 1 & 1 & Metallurgia & 2 & - \\
Commercio & - & 2 & Servizi & - & 1 \\
Logistica & 1 & - & Logistica & - & 1 \\
Severstal & - & - & Commercio & - & 5 \\
Energia & 1 & - & Plasturgia & 1 & - \\
Meccanica & 1 & - & Carta & 1 & - \\
Metallurgia & 3 & - & Aerospazio & - & 1 \\
Servizi & 1 & - & Holding industriale & 1 & - \\
Logistica & 1 & - & Totale & 30 & 11 \\
\hline
\end{tabular}

Fonte: nostre elaborazioni su dati Invitalia.

Renova è uno dei principali gruppi conglomerati russi, con prevalente attività in ambito metallurgico ed energetico. Tra le operazioni più interes-

22. Le altre imprese sono collocate in Piemonte (3) e Toscana (3), Lazio (2) e Puglia (2), Sardegna, Friuli Venezia Giulia e Sicilia (1 impresa ciascuna). 
santi realizzate in Italia vi è l'acquisizione di Energetic Source, nel comparto dell'energia elettrica (Vegezzi, 2008) e di Kerself, legata alla progettazione e produzione di impianti fotovoltaici (Vegezzi, 2008; Giliberto, 2007).

Severstal, specializzato nei settori metallurgico ed estrattivo, ha acquisito Lucchini spa e Radaelli Tecna spa, imprese di eccellenza in produzioni speciali di acciaio. Finalità market seeking ed efficiency seeking per una estensione del controllo all'estero delle fasi a monte e a valle della propria filiera produttiva emergono nettamente in queste iniziative (Festa, 2005; Scott, 2009). Con analoghe motivazioni strategiche, Evraz, uno dei player nel settore minerario a livello globale, ha acquisito Palini \& Bertoli spa, azienda leader nella produzione di lamiere da treno "quarto" in acciaio.

Altro protagonista degli investimenti russi in Italia è Rusal, maggior produttore mondiale di alluminio (Unctad, 2007), che ha âcquisito in Sardegna impianti di estrazione di alluminia (Festa, 2006 c). Novolipetsk, secondo gruppo siderurgico russo, ha realizzato accordi sempre nel settore dell'acciaio sia con joint venture sia mediante acquisizioni di aziende italiane, tra cui in particolare le Acciaierie Grigoli (Festa, 2006a e b).

Ulteriore importante operazione ha coinvolto Lukoil, uno dei principali player mondiali nel settore del petrolio e del gâs, per l'investimento, in Sicilia, in una delle più grandi raffinerie del Mediterraneo (D'Ascenzo, 2008; Giliberto, 2009).

Anche in Italia è rilevante, inoltre, la presenza di Gazprom, che ha siglato joint venture ed accordi, in particolare con Eni, volti ad acquisire il controllo dell'intera filiera legata al gas: dall'estrazione alla distribuzione (Giliberto, 2006; Kheifets, 2008).

Infine, motivazioni legate al mercato ed alla ricerca di risorse ad alto valore aggiunto hanno caratterizzato l'accordo in joint venture tra Sukhoi Company, maggiore gruppo aeronautico russo, ed Alenia Aeronautica, società di Finmeccanica, per la realizzazione di attività commerciali sui mercati occidentali di un velivolo da trasporto regionale (Pasqualetto, 2007).

Nel complesso, dunque, il contesto italiano presenta molte analogie, in termini di trend e modalità di investimento, rispetto all'attività delle Mne russe riscontrabile a livello globale. Pur non possedendo l'Italia vantaggi e posizioni competitive rilevanti nel settore primario, gli investitori russi hanno comunque trovato spazi per realizzare investimenti strategici nei settori metallurgico ed energetico, con riferimento non solo alle attività estrattive e produttive, ma anche in termini di poli/attività logistici e distributivi. Sono stati acquisiti domini in varie aree del Paese che dovrebbero consentire alle imprese russe di assumere posizioni strategiche nel Mediterraneo e nei Balcani. Parallelamente, sono state realizzate negli ultimi mesi alcune 
operazioni di acquisizione in comparti dei servizi e manifatturieri, soprattutto nella meccanica, e si sono affacciati investitori nuovi.

In termini prospettici l'Italia potrebbe divenire, anche in virtù delle intense relazioni politiche esistenti tra i governi italiano e russo, un partner rilevante ed una destinazione privilegiata per gli investitori russi, tanto più quanto risulteranno coinvolte nelle operazioni transnazionali anche le imprese di dimensioni medie e di settori diversi dal primario. Queste ultime, molto più dei gruppi statali o delle grandi Mne, potrebbero trovare un fertile terreno in Italia per alleanze ed acquisizioni. Fattori rilevanti per la scelta di investimenti nel Paese potrebbero essere rappresentati sia dalla presenza di risorse immateriali ad alto valore aggiunto, fondamentali per l'affermazione nei mercati occidentali (conoscenze specialistiche, immagine, marchi in primis), sia la disponibilità di imprese target di dimensioni ridotte, finanziariamente "accessibili".

\section{Alcune considerazioni di sintesi}

Il quadro degli investimenti russi, appena delineato, stimola alcune considerazioni, da un lato, sul ruolo che gli Ide potrebbero avere per lo sviluppo del Paese e, dall'altro, sulle criticità che li connotata.

In questo momento storico gli investimenti diretti esteri rappresentano una leva cruciale di sostegno allo sviluppo della Federazione. La crisi finanziaria ha messo, infatti, in evidenza le forti debolezze dell'economia russa (Oecd, 2009) ${ }^{23}$, che è strettamente dipendente dalle esportazioni di materie prime - petrolio e gas naturale ne assorbono oltre il $60 \%$ - ed in generale dai prezzi delle materie prime (Hamilton, 2005; Golubchikov, 2007; Connolly, 2009; The Economist, 2009a). Gli analisti sottolineano come l'economia debba trasformarsi, evolvendo da un modello basato sull'industria primaria ad uno legato maggiormente ai servizi ed all'innovazione. Occorre potenziare i settori manifatturieri tradizionali, dalla meccanica e componentistica, all'elettronica, all'auto, alla farmaceutica, in cui il livello di competitività è tuttora molto basso in raffronto alle imprese occidentali (Ahrend, 2006).

23. Nel contesto economico globale la Russia sta realizzando le performance peggiori rispetto alle altre economie emergenti, con un calo del Pil dell' $8,5 \%$ a fronte della crescita della Cina di oltre 1' $8 \%$, disoccupazione a livelli superiori all' $8 \%$ e produzione industriale in calo del 9,5\% su base annua (Connolly, 2009; World Bank, 2009). Anche le stime di crescita sono pessimiste: i livelli attesi per il 2010 sono del 3,1\%, a fronte del 9\% Cinese (The Economist, 2009b). I ritmi di crescita pre crisi ( $8,7 \%$ annuo) dovrebbero riaffermarsi solo dopo il 2012 (Kramer, 2009). 
Gli Ide possono rappresentare uno strumento di crescita rapida ed un'opportunità di maturazione per le imprese russe. Il tutto a fronte della forte concorrenza internazionale che diventerà ancora più pressante in caso di ingresso della Russia nel Wto (Jensen et al., 2007). Le acquisizioni, in particolare, possono consentire sia di aver accesso ad asset strategici, mercati di sbocco e nuove competenze, sia di importare modelli gestionali innovativi. Tutte risorse, queste, necessarie per una maggiore integrazione e competitività internazionale.

Numerosi sono, tuttavia, gli aspetti critici da affrontare affinché la leva degli Ide possa efficacemente supportare la crescita del Paese.

Vi è, innanzitutto, la necessità di superare la forte resistenza che molto spesso gli investimenti riscontrano in occidente, per le ingerenze effettive o presunte del governo nelle singole operazioni (Sauvant, 2005). Interessi economici e geo-politici tendono talvolta a sovrapporsi, fâcendo fallire o rendendo complesse le trattative.

Non esistono, inoltre, a tutt'oggi una regolamentazione specifica per gli investimenti esteri e misure di sostegno particolari ai processi di internazionalizzazione (Sauvant, 2005; Kheifets, 2008). A fronte delle grosse operazioni realizzate dai colossi del settore primario, per le imprese private e di dimensioni medie si riscontrano fortissime difficoltà. Ridotte risorse finanziarie a disposizione, scarsa conoscenza dei mercati esteri e delle pratiche manageriali occidentali, mancanza di reti di business internazionali sono solo alcuni dei problemi riscontrati (Kheifets, 2008).

L'urgenza di avviare una vera e propria politica di stimolo degli investimenti, come fatto dalla Cina attraverso la strategia del Go Global, è riconosciuta dalle Autorità russe (Belton, 2008). Una regolamentazione specifica degli investimenti stessi, unitamente ad un sistema di incentivi e supporti alle imprese, darebbero un segnale univoco ai Paesi ospitanti circa l'efficienza e la trasparenza delle iniziative russe. Questo con un impatto positivo di immagine anche per attrarre flussi di investimento in entrata (Kheifets, 2008). Si stimolerebbero, inoltre, le imprese domestiche, dei settori emergenti e anche di dimensioni minori, ad espandersi verso i Paesi occidentali (Sauvant, 2005; Belton, 2008; Kheifets, 2008; Levy, 2009). Si avrebbe, in tal modo, la possibilità di passare da una focalizzazione esclusiva verso investimenti volti a sfruttare vantaggi posseduti, come quelli dalle imprese del settore primario, ad iniziative finalizzate ad acquisire, internalizzare e valorizzare nuovi assett (Cantwell, 1989, 1995; Kuemmerle, 1999; Dunning e Narula, 1996). Una politica mirata ed attiva di internazionalizzazione potrebbe, infine, supportare lo sviluppo di settori evoluti ed alta intensità di tecnologia in cui la Russia presenta degli elevati potenziali, per il possesso di risorse distintive, soprattutto in termini di know how e compe- 
tenze specialistiche delle risorse umane (Bardhan e Kroll, 2006) ${ }^{24}$. Il tutto a supporto del processo di cambiamento, richiamato, dell'economia russa.

Il quadro delineato dall'analisi consente di formulare alcune riflessioni utili ad alimentare il percorso di ricerca in cui si inserisce questo lavoro.

Come si è accennato in apertura, tale percorso mira, innanzitutto, a contribuire al dibattito scientifico in corso sulla opportunità di adeguamento delle più consolidate teorie sui flussi di investimento alla luce del nuovo ruolo assunto dalle economie emergenti quali investitori globali. Le tipicità riscontrate nell'attività di investimento della Federazione e nella posizione complessiva del Paese su scala internazionale giustificano sicuramente una rilettura di alcune di queste teorie. A livello comparativo, rispetto alle altre economie dell'area Bric, il caso russo appare interessante sia per il tipo di iniziative realizzate - di ampia portata finanziaria e fortemente concentrate a livello settoriale - sia per il ricorso, da parte delle imprese, allo strumento delle acquisizioni sin dalle fasi iniziali del processo di apertura internazionale. Altra peculiarità riguarda il fatto che gli investimenti sono indirizzati prevalentemente nei Paesi industrializzati.

Sempre rispetto alle finalità del percorso di ricerca di lungo termine richiamato, focalizzando l'attenzione sul caso italiano, appare interessante approfondire ulteriormente l'analisi dell'impatto degli investimenti russi sul sistema produttivo nazionale in almeno tre ambiti: infrastrutture logistiche, meccanica, energia.

Con riferimento agli asset logistici ed infrastrutturali, seppure in Italia siano solamente tre i progetti realizzati, ci si potrebbe attendere una espansione degli stessi, sulla scia del trend riscontrato a livello mondiale ed in Europa in particolare. Si tratta di un settore da monitorare per la strategicità che lo stesso assume ai fini della competitività del Paese e per il forte interesse che altri operatori, sempre legati ad economie emergenti, stanno mostrando. Il caso della Cina, in questo senso, è emblematico (Spigarelli, 2009).

In relazione alla meccanica, invece, la crescita dei flussi di Ide ed il numero di imprese a controllo russo giustificano un ulteriore sforzo di analisi per comprendere l'impatto che la presenza russa sta avendo e potrebbe avere, in prospettiva, sul tessuto produttivo nazionale. Il settore della meccanica è ricco di aziende con vantaggi distintivi, basati su conoscenze e know how, oltre che su legami e rapporti di partnership formali ed informali

24. Si pensi, ad esempio, al settore del software. In esso potrebbe trovare utile riconversione il personale fuoriuscito dall'industria bellica o dagli enti di ricerca pubblici, interessati negli ultimi anni da processi di ridimensionamento e razionalizzazione. Le elevate qualifiche professionali e l'alto profilo formativo posseduti rappresentano ad oggi un asset non sfruttato e dalle grosse potenzialità (Bardhan e Kroll, 2006). 
nell'ambito delle reti locali. In questo senso, appare utile approfondire lo studio per comprendere se ed in che modo, inserendosi nella filiera produttiva, gli investitori russi stiano influenzando i pattern evolutivi dei sistemi locali e delle aree distrettuali. Il fatto che si tratti di imprese di grandi dimensioni che agiscono in prevalenza mediante acquisizioni, rende l'analisi particolarmente interessante. Ciò in relazione sia al potere contrattuale che le stesse potrebbero mostrare verso fornitori e subfornitori, sia all'eventuale capacità di estendere progressivamente il controllo sulla filiera produttiva.

Riguardo al settore energetico, infine, ciò che emerge è l'investimento dei grossi gruppi conglomerati russi in piccole imprese italiane, legate al settore della produzione dell'energia rinnovabile o degli impianti/attrezzature ad essa collegati. L'interesse di tali colossi in una nicchia così particolare appare degna di approfondimenti per comprenderne, innanzitutto, le motivazioni strategiche sottostanti. Si tratta di settori sûssidiati, in cui l'investitore potrebbe cercare di cogliere i benefici di un sostegno esterno della domanda. Tuttavia, l'impatto atteso sulla redditività di gruppo potrebbe non giustificare investimenti diretti in segmenti di business tanto specifici. Si potrebbe trattare, piuttosto, della ricerca di asset strategici nelle imprese acquisite, in termini di conoscenze tecniche e know how. Oppure ancora le acquisizioni potrebbero rientrare in una logica più ampia di accordi con partner italiani coinvolti nel settore energetico. Altrettanto interessante appare monitorare il trend delle iniziative e l'impatto che le stesse potrebbero avere, in caso di loro ulteriore espansione, sul settore energetico nazionale.

Ricevuto: 8 gennaio 2010 - Accettato: 2 settembre 2010

\section{Sommario}

Gli investimenti diretti esteri della Federazione russa

Gli investimenti diretti all'estero stanno caratterizzando l'espansione internazionale di molte economie emergenti, che fanno leva su di essi per affermarsi rapidamente a livello economico e politico globale.

La Russia è uno dei protagonisti in questo scenario. Essa si contraddistingue sia per il fatto che le imprese investitrici all'estero sono poche ed operanti tipicamente nel settore primario, sia per la portata finanziaria delle singole iniziative. Il lavoro propone un'analisi delle principali caratteristiche del fenomeno, a livello globale, europeo ed italiano: direttrici degli investimenti, trend principali, protagonisti e motivazioni strategiche sottostanti. Alcune riflessioni di sintesi su criticità e prospettive del processo di internazionalizzazione dell'economia russa chiudono il contributo. 


\begin{abstract}
Outward Foreign Direct Investments of the Russian Federation

Outward foreign direct investments are becoming a key lever in the global political and economic affirmation of many emerging economies. The Russian Federation is an interesting example of this phenomenon. Its global expansion is characterized by two aspects. First, the main players in foreign expansion are a few companies operating in the primary sector. Second, each single initiative involves huge financial resources. The paper proposes an analysis of the main features of the international investment activity of the Russian Federation, at the global, European and Italian level. The main trends in investments and players are analysed, as well as the strategic motivations behind the initiatives. The paper closes with examination of the critical aspects and perspectives involved in the Russian internationalization process.
\end{abstract}

EconLit Classification: F210, F230, P330

Keywords: Russian outward foreign direct investments, Russian multinational enterprises, Russian owned Italian enterprises

Parole chiave: Investimenti diretti esteri russi, Multinazionali russe, Imprese italiane a capitale russo

\title{
Riferimenti bibliografici
}

Ahrend R. (2006), «Russian Industrial Restructuring: Trends in Productivity, Competitiveness and Comparative Advantage», Post-Communist Economies, 18, 3, pp. 277-295.

Aykut D., Goldstein A. (2006), «Developing Country Multinationals: South-South Investment Comes of Age», Oecd Development Centre Working Papers, 257, Oecd Development Centre.

Andreff W. (2002), «The new multinational corporations from transition countries», Economic Systems, 26, 4, pp. 371-379.

Andreff W. (2003), «The Newtransnational Corporations: Outward Foreign Direct Investment from Post-Communist Economies in Transition», Transnational Corporations, 12, 2, pp. 73-118.

Aton corporation (2004), Russian Market 2005, 4 November, Aton Capital Group, Mosca.

Bardhan A.D., Kroll C. (2006), «Competitiveness and an Emerging Sector: The Russian Software Industry and its Global Linkages», Industry \& Innovation, 13, 1, pp. 69-95.

Bellabona P., Spigarelli F. (2006), «Go Global: le politiche di internazionalizzazione attiva della Cina», Economia Marche, 3, pp. 185-204.

Bellabona P., Spigarelli F. (2007), «Moving from Open Door to Go Global: China goes on the world stage», International Journal on Chinese Culture and Management, 1, pp. 93-107. 
Belton C. (2008), «Copy China and Invest Abroad, Says Medvedev», Financial Times, 1st February.

Bertoni F., Elia S., Rabbiosi L. (2008), «Drivers of Acquisitions from BRICs to Advanced Countries: Firm-Level Evidence», "Emerging Multinationals: Outward Foreign Direct Investment from Emerging and Developing Economies", Copenhagen, 9-10th October; Available on linedex.dk/ofdi/32\%20Bertoni $\% 20$ Fabio.pdf (last access: 15th July).

Bevan A., Estrin S. (2004), «The Determinants of Foreign Direct Investment into European Transition Economies», Journal of Comparative Economics, 32, pp. 775-787.

Boffa F., Galeazzi G., Spigarelli F. (2008), «Recenti tendenze nei flussi di investimento estero delle economie emergenti. Sovereign Wealth Funds, imprese globali ed effetti per i Paesi sviluppati», Economia Marche, 2, pp. 117-148.

Bonaglia F., Goldstein A. (2008), Globalizzazione e sviluppo, il Mulino, Bologna.

Bonaglia F., Goldstein A., Mathews J.A. (2007), «Accelerated Internationalization by Emerging Markets' Multinationals: The Case of the White Goods Sector», Journal of World Business, 42, 4, pp. 369-383.

Braguinsky S., Myerson R. (2007), «A Macroeconomic Model of Russian Transition», Economics of Transition, 15, 1, pp. 77-107.

Buckley P. (2002), «Is the International Business Research Agenda Running out of Steam?», Journal of International Business Studies, 33, pp. 365-373.

Bulatov A. (1998), «Russian Direct Investment Abroad: Main Motivations in the Post-Soviet Period», Transnational Corporations, 7, 1, pp. 69-82.

Bulatov A. (2001), «Russian Direct Investment Abroad: History, Motives, Finance, Control and Planning», Economics of Planning, 34, 3, pp. 179-194.

Cantwell J. (1989), Technological Innovation and Multinational Corporations, Basil Blackwell, Oxford.

Cantwell J. (1995), «The Globalisation of Technology: What Remains of the Product Cycle Model?», Cambridge Journal of Economics, 19, pp. 155-174.

Cantwell J., Barnard H. (2008), «Do Firms from Emerging Markets Have to Invest Abroad? Outward FDI and the Competitiveness of Firms», in Sauvant K.P. (ed.) The Rise of Transnational Corporations from Emerging Markets: Threat or Opportunity?, Edward Elgar Publishing, Willinston, pp. 55-85.

Connolly R. (2009), «Russia and the Economic Crisis», Russian Analytical Digest, 65, Available on line: www.res.ethz.ch. (last access: 30th December 2009).

Cuervo-Cazurra A., Genc M. (2008), «Transforming Disadvantages into Advantages: Developing-Country Mnes in the Least Developed Countries», Journal of International Business Studies, 39, 6, pp. 957-979.

D’Ascenzo M. (2008), «Più utili per Erg e Lukoil», Il Sole 24 Ore, Mercoledì 25 Giugno, p. 7.

Dunning J.H. (1977), «Trade, Location of Economic Activity and the Multinational Enterprise: A Search for an Eclectic Approach», in Ohlin B., Hesselborn P.O., Wijkman P.E. (eds.), The International Allocation of Economic Activity, Macmillan, London, pp. 395-418.

Dunning J.H. (1981), «Explaining the International Position of Countries Towards 
a Dynamic or Developmental Approach», Weltwirtschaftliches Archiv, 117, pp. 30-64.

Dunning J.H. (1986), «The Investment Development Path Revisited», Weltwirtschaftliches Archiv, 122, pp. 667-677.

Dunning J.H. (1988), «The Eclectic Paradigm of International Production: A Restatement and Some Possible Extensions», Journal of International Business Studies, 19, 1, pp. 1-31.

Dunning J.H. (1993), Multinational Enterprises and the Global Economy, Wokingham, Addison Wesley, England.

Dunning J.H., Narula R. (1996), «The Investment Development Path Revisited Some Emerging Issues», in Dunning J.H., Narula R. (eds.), Foreign Direct Investment and Governments, Routledge, London, pp. 350-390.

Dunning J.H., Kim C., Park D. (2008), «Old Wine in New Bottles: A Comparison of Emerging Market Tncs Today and Developed Country Tncs Thirty Years Ago», in Sauvant K.P. (ed.), The Rise of Transnational Corporations from Emerging Markets: Threat or Opportunity?, Edward Elgar Publishing, Willinston, pp. 158-180.

Dunning J.H., Lundan S.M. (2008), Multinational Enterprises and the Global Economy, II ed., Edward Elgar, Cheltenham.

Elenkov D.S. (1995a), «Russian Aerospace MNCs in Global Competition: Their Origin, Competitive Strengths and Forms of Multinational Expansion», The Columbia Journal of World Business, 30, 2, pp. 66-78.

Elenkov D.S. (1995b), «The Russian Aerospace Industry: Survey with Implications for American Firms in the Global Marketplace», Journal of International Marketing, 3, 2, pp. 69-79.

Eurostat (2008), European Union Foreign Direct Investment Yearbook 2008, Available on line: http://epp.eurostat.ec.europa.eu/cache/ITY OFFPUB/KSBK-08-001/EN/KS-BK-08-001-EN.PDF (last access: 30th December 2009).

Federico S., Minerva G.A. (2007), «Outward FDI and Local Employment Growth in Italy», Temi di Discussione del Servizio Studi, Economic Working Papers, 613 , Banca d'Italia.

Festa C. (2005), «Severstal conquista Lucchini», Il Sole 24 Ore, 8 febbraio, p. 33.

Festa C. (2006a), «Un partner russo per Duferco», Il Sole 24 Ore, 19 ottobre, p. 20.

Festa C. (2006b), «Nell' acciaio altri accordi», Il Sole 24 Ore, 21 ottobre, p. 10.

Festa C. (2006c), «La Videocon riprova in Italia Benelli prima preda dei cinesi», Il Sole 24 Ore, 11 novembre, p. 15.

Gammeltoft P. (2008), «Emerging Multinationals: Outward FDI from the BRICS Countries», International Journal of Technology and Globalisation, 4, 1, pp. 5-22.

Giliberto J. (2006), «Eni più forte in Russia, Gazprom in Italia», Il Sole 24 Ore, 15 novembre, p. 3.

Giliberto J. (2007), «Vekselberg nell'impianto di San Severo», Il Sole 24 Ore, 16 novembre, p. 5.

Giliberto, J. (2009), «La russa Lukoil pronta a entrare nel mercato del gas», Il Sole 24 Ore, 3 giugno, p. 17.

Goldstein A. (2009), Multinational Companies from Emerging Economies. Com- 
position, Conceptualization and Direction in the Global Economy, Palgrave Macmillan, Hampshire.

Goldstein A., Pusterla F. (2008), «Emerging Economies’ Multinationals: General Features and Specificities of the Brazilian and Chinese Cases», Cespri - Centro di Ricerca sui Processi di Innovazione e Internazionalizzazione, Università Commerciale "Luigi Bocconi”, Working Paper, 228.

Golubchikov O. (2007), «Re-Scaling the Debate on Russian Economic Growth: Regional Restructuring and Development Asynchronies», Europe-Asia Studies, 59, 2, pp. 191-215.

Guille'n M. F., Garci'a-Canal E. (2009), «The American Model of the Multinational Firm and the "New" Multinationals from Emerging Economies», Academy of Management Perspectives, May, pp. 23-35.

Gusev K.N. (2004), «Size and Structure of Russian Investments Abroad», Bankovskoe delo, 12th December, Available on line: http://www.bankdelo.ru/ archive/nomer1204/index.php?page=2 (last access: 30th December 2009).

Hamilton C.B. (2005), «Russia's Economic Integration. Escapism and realities», Economic Systems, 29, pp. 294-306.

Heckscher E.F. (1919), «The Effect of Foreign Trade on the Distribution of Income», Ekonomisk Tidskrift, pp. 497-512, tradotto in Flam H., Flanders M.J. (eds.) (1991), Heckscher-Ohlin Trade Theory, Mit Press, Cambridge, pp. 497-512.

Heinrich A. (2001), «Internationalisation, Market Structures and Enterprise Behaviour: The Janus-Faced Russian Gas Monopoly Gazprom», in Liuhto K. (eds.), East Goes West: The Internationalization of Eastern Enterprises, Lappeenranta University of Technology, Lappeenranta, pp. 51-87.

Heinrich A. (2003), «Internationalisation of Russia's Gazprom», Journal of East European Management Studies, 8, 1, pp. 46-66.

Heinrich A. (2005), «Between a Rock and a Hard Place: The Energy Sector in Central and Eastern Europe», in Liuhto K., Vincze Z. (eds.), Wider Europe, Turku School of Economics and Business Administration, Turku, pp. 457-490.

Jensen J., Rutherford T., Tarr D. (2007), «The Impact of Liberalizing Barriers to Foreign Direct Investment in Services: The Case of Russian Accession to the World Trade Organization», Review of Development Economics, 11, 3, pp. 482-506.

Johanson J., Vahlne J.E. (1977), «The Internationalization Process of the Firm. A Model of Knowledge Development and Increasing Market Commitments», Journal of International Business Studies, 8, 1, pp. 23-32.

Johanson J., Vahlne J.E. (1990), «The Mechanism of Internationalisation», International Marketing Review, 7, 4, pp. 11-24.

Johanson J., Wiedersheim-Paul F. (1975), «The Internationalization of the Firm. Four Swedish Case Studies», Journal of Management Studies, 12, 3, pp. 305-322.

Kalotay K. (2001), «The Contribution of Foreign Direct Investment to Transition Revisited», The Journal of World Investment, 2, 2, pp. 259-276.

Kalotay K. (2002), «Outward Foreign Direct Investment and Governments in Central and Eastern Europe: The Cases of the Russian Federation Hungary and Slovenia», The Journal of World Investment, 3, 2, pp. 267-287.

Kalotay K. (2003), «Outward FDI from Economies in Transition in a Global Con- 
text», Journal for East European Management Studies, 8, 1, pp. 6-24.

Kalotay K. (2005), «Outward Foreign Direct Investment from Russia in a Global Context», Journal of East-West Business, 11, 3-4, pp. 9-22.

Kalotay K. (2008a), «Russian Transnationals and International Investment Paradigms», Research in International Business and Finance, 22, 2, pp. 85-107.

Kalotay K. (2008b), «How to Explain the Foreign Expansion of Russian Firms», Journal of Financial Transformation, 28, pp. 53-61.

Kets de Vries M., Shekshnia S., Korotov K., Florent-Treacy E. (2004), «The New Global Russian Business Leaders: Lessons from a Decade of Transition», European Management Journal, 22, 6, pp. 637-648.

Kheifets B. (2008), «The External Sector of the Russian Economy», Problems of Economic Transition, 51, 4, pp. 63-80.

Kramer A. (2009), «Russia Facing Long Recession, World Bank Says», The New York Times, 25th June.

Kuemmerle W. (1999), «Foreign Direct Investment in Industrial Research in the Pharmaceutical and Electronic Industries - Results from a Survey of Multinational Firms», Research Policy, 28, 2-3, pp. 179-193.

Kuznetsov A. (2008), «Russian Business Expansion», Russian Analytical Digest, 34, Available in line: www.res.ethz.ch (last access: 30th December 2009).

Levy C. (2009), «Russia's Leaders See China as Template for Ruling», The New York Times, 18th October.

Li S., Tallman S.B., Ferreira M.P. (2005), «Developing the Eclectic Paradigm to a Model of Global Strategy: An Application to the Impact of Sep 11 Terrorist Attacks on Mne Performance», Journal of International Management, 11, 4, pp. 479-496.

Lipsey R.E. (2001), «Foreign Dirêct Investment and the Operations of Multinational Firms: Concepts, History and Data», Nber Working Paper, 8665.

Liuhto K. (2001a), «Russian Gas and Oil Giant Conquer Markets in the West: Evidence on the Internationalization of Gazprom and LUKoil», Journal of EastWest Business, 7, 3, pp. 31-72.

Liuhto K. (ed.) (2001b), East Goes West: The Internationalization of Eastern Enterprises, Lappeenranta University of Technology, Lappeenranta.

Liuhto K. (ed.) (2005), Expansion or Exodus: Why Do Russian Corporations Invest Abroad?, Haworth Press, Binghamton, Ny.

Liuhto K. (2007), «A Future Role of Foreign Firms in Russia's Strategic Industries», Electronic Publications of Pan-European Institute, 4.

Liuhto K., Jumpponen J. (2003), «The Russian Eagle Has Landed Abroad: Evidence Concerning the Foreign Operations of the Russian Federation's 100 Biggest Exporters and Banks», Research Report,141, Lappeenranta University of Technology, Lappeenranta.

Locatelli C. (2006), «The Russian Oil Industry Between Public and Private Governance: Obstacles to International Oil Companies' Investment Strategies», Energy Policy, 34, 9, pp. 1075-1085.

Mathews J.A. (2002), Dragon Multinationals: A New Model of Global Growth, Oxford University Press, New York. 
Mathews J.A. (2006), «Dragon Multinationals: New Players in 21st Century Globalization», Asia Pacific Journal of Management, 23, 1, pp. 5-27.

Meyer K., Nguyen H.V. (2005), «Foreign Investment Strategies and Sub-National Institutions in Emerging Markets: Evidence from Vietnam», Journal of Management Studies, 42, 1, pp. 63-93.

Moon H.C., Rohel T.W. (1993), «An Imbalance Theory of Foreign Direct Investments», Multinational Business Review, spring, pp. 56-65.

Moon H.C., Rohel T.W. (2001), «Unconventional Foreign Direct Investment and the Imbalance Theory», International Business Review, 10, 2, April, pp. 197215.

Morck R., Yeung B., Zhao M. (2008), «Perspectives on China’s Outward Foreign Direct Investment», Journal of International Business Studies, 39, 3, pp. 337-50.

Mori A., Rolli V. (1998), «Investimenti diretti all'estero e commercio: complementi o sostituti?», Temi di discussione, 337, Banca d'Italia, Roma.

North D.C. (1990), Institutions, Institutional change and Economic Performance, Cambridge University Press, Cambridge, Massachusetts.

Oecd (2008), Oecd Investment Policy Reviews: China 2008 Encouraging Responsible Business Conduct, Available on line: http://www.oecd.org/document/ 40/0,3343, en_2649_34893_41735656_1_1_1_1,00\&\&en-USS_01DBC.html (last access: 15 July 2010).

Oecd (2009), Economic Surveys: Russian Federation, July, 6, Oecd.

Ohlin B. (1933), Interregional and International Trade, Harvard University Press, Cambridge, Ma.

Panibratov A., Kalotay K. (2009), «Russian Outward FDI and its Policy Context», Columbia FDI Profiles. Country profiles of inward and outward foreign direct investment, Vale Columbia Center on Sustainable International Investment, $\mathrm{n}$. 1, 13th October, Available on line: http://www.vcc.columbia.edu/documents/ FDIProfile-Russia.pdf (last access: 30th December 2009).

Pasqualetto C. (2007), «Parte a Venezia la joint Alenia-Sukhoi», Il Sole 24 Ore, 11 settembre, p. 35.

Pelto E., Vahtra P., Liuhto K. (2003), «Cyp-Rus Investment Flows to Central and Eastern Europe-Russia's Direct and Indirect Investments via Cyprus to CEE», Electronic Publications of Pan European Institute, Turku School of Economics and Business Administration, 2.

Peng M.W. (2002), "Towards an Institution-Based View of Business Strategy". Asian Pacific Journal of Management, 19, 2, pp. 251-267.

Petridou K. (2008), «EU-27 Foreign Direct Investments in Brazil, Russia, India and China Increased by More than 50\% in 2007», Statistics in focus, Economy and finance, 64, Eurostat, Available on line: http://epp.eurostat.ec.europa.eu/ cache/ITY OFFPUB/KS-SF-08-064/EN/KS-SF-08-064-EN.PDF (last access: 30th December 2009).

Ramamurti R. (2008), What Have We Learned About Emerging Market Mnes?, Cambridge University Press, Cambridge.

Ramamurti R., Singh J. (eds.) (2009), Emerging Multinationals in Emerging Markets, Cambridge University Press, Cambridge. 
Rugman A. (2008), «How Global are TNCs from Emerging Markets?», in Sauvant K.P. (ed.), The Rise of Transnational Corporations from Emerging Markets: Threat or Opportunity?, Edward Elgar, Cheltenham, pp. 86-106.

Samuelson P. (1948), «International Trade and the Equalization of Factor Prices», Economic Journal, 58, pp. 163-184.

Samuelson P. (1949), «International Factor-Price Equalisation Once Again», Economic Journal, 59, pp. 181-197.

Sauvant, K.P. (2005), «New Sources of FDI: the BRICs. Outward FDI from Brazil, Russia, India and China», The Journal of World Investment \& Trade, 6, pp. 640-647.

Sauvant K.P. (2008), «The Rise of Transnational Corporations from Emerging Markets: The Issues», in Sauvant K.P. (ed.), The Rise of Transnational Corporations from Emerging Markets: Threat or Opportunity?, Edward Elgar, Cheltenham, pp. 3-14.

Scott A. (2009), «Mosca sposta i confini dell'impero», Il Sole 24 Ore, 31 maggio, p. 3.

Scott W.R. (2002), «The Changing World of Chinese Enterprises: An Institutional Perspective», in Tsui A.S., Lau M. (eds.), Management of Enterprises in People's Republic of China, Kluwer Academic Press, Boston, pp. 59-78.

Settles A. (2009), Corporate Social Responsibility Impact on Foreign Direct Investment Practices of Russian Multinational Corporations: Do MNCs with Better CSR Practices Have Lower Cost of OFDI?, Available on line: ww.hse.ru/data/2009/ 10/14/1238606508/FDICSR10052009sht.doc (last access: 15th July 2010).

Shekshnia S. (2001), «Troika Dialog Founder Ruben Vardanian on Building Russia's First Investment Bank», Academy of Management Executive, 15, 4, pp. 16-23.

Spigarelli F. (2009), «Multinazionåli dai Paesi emergenti: il caso delle imprese cinesi», Economia e Politica Industriale, 2, pp. 131-159.

Spigarelli F. (in corso di pubblicazione), «L'espansione internazionale delle imprese russe: il caso italiano», in Beretta S., Pissavino P. (a cura di), Russia e oltre. Relazioni economiche ed equilibri politici. In ricordo di Vittorio BeonioBrocchieri, Rubbettino, Soveria Mannelli.

Stecco M. (2008), Investimenti russi in Italia: realtà recente e prospettiva importante, pp. 86-97, testo disponibile al sito: http://www.italplanet.it/FilePub/ 42689279286-87.pdf (ultimo accesso: 30 dicembre 2009).

The Economist (2009a), «A New Sick Man», The Economist, 4th June, 391, 8634, pp. 22-25.

The Economist (2009b), «Not Just Straw Men», The Economist, 18th June, 392, 8636, pp. 63-65.

Uctad (2005), Case Study on Outward Foreign Direct Investment by Russian Enterprises, Available on line: www.unctad.org/en/docs/c3em26d2a4_en.pdf (last access: 30th December 2009).

Unctad (2006), World Investment Report. Overview FDI from Developing and Transition Economies, United Nations, New York and Geneva.

Unctad (2007), World Investment Report 2007, United Nations, New York and Geneva. 
Unctad (2009a), World Investment Report 2009, United Nations, New York and Geneva.

Unctad (2009b), Country Fact Sheet: Russian Federation, Available on line: http://www.unctad.org/sections/dite_dir/docs/wir08_fs_ru_en.pdf (last access: 30th December 2009).

Vahtra P., Liuhto K. (2004), Expansion or Exodus? Foreign Operations of Russia's Largest Corporations, Lappeenranta University of Technology, Lappeenranta.

Valdani E., Bertoli G. (2006), Mercati internazionali e marketing, Egea, Milano.

Vegezzi G. (2008), «Italia, porta d'ingresso per l'Europa», Il Sole 24 Ore, 18 luglio, p. 36.

World Bank (2009), «The World Bank in Russia», Russian Economic Report, 19.

Wright M., Filatotchev I., Hoskisson R.E., Peng M.W. (2005), «Strategy Research in Emerging Economies: Challenging the Conventional Wisdom», Journal of Management Studies, 42, 1, pp. 1-33.

Yamakawa Y., Peng M., Deeds D. (2008), «What Drives New Ventures to Internationalize from Emerging to Developed Economies?», Entrepreneurship Theory and Practice, 32, 1, pp. 59-82. 